\title{
Stable finite volume element schemes for the shallow-ice approximation
}

\author{
ED BUELER
}

\begin{abstract}
Department of Mathematics and Statistics and Geophysical Institute, University of Alaska Fairbanks, Fairbanks, AK, USA Correspondence: Ed Bueler <elbueler@alaska.edu>
\end{abstract}

\begin{abstract}
The isothermal, non-sliding shallow-ice approximation, combined with mass conservation, is a fundamental model for ice-sheet and glacier flow. It determines the ice extent, geometry and velocity by the solution of a free-boundary problem. In this paper, the steady-state form of this problem is solved directly, without time-stepping, thereby demonstrating a fully implicit scheme with no stability restrictions. The classical Mahaffy (1976) finite difference method is first reinterpreted as a 'finite volume element' scheme that has both an everywhere-defined approximate thickness function and an approximation of the conservation equation in flux integral form. From this reinterpretation an improved scheme is built by using better quadrature in the integral and upwinding on that part of the flux which is proportional to the bed gradient. The discrete equations are then solved by a parallel Newton scheme which respects the constraint that ice thickness is non-negative. The results show good accuracy on both flat-bed and bedrock-step exact solutions. The method is then applied at high resolution to model the steady-state geometry of the Greenland ice sheet, using only bedrock elevation and present-day surface mass balance as input data.
\end{abstract}

KEYWORDS: glacier modelling, ice-sheet modelling, free boundary, mass conservation, bedrock topography

\section{INTRODUCTION}

The first successful numerical approach to modeling ice-sheet flow and geometry evolution in two horizontal dimensions was the classical finite difference (FD) scheme introduced by Mahaffy (1976). This scheme numerically solves the shallow-ice approximation (SIA; Hutter, 1983) by computing the ice flux at staggered-grid points using particular choices when evaluating the ice thickness and surface slope. An advantage of the scheme is its relatively small stencil, which reduces memory access in implicit or semi-implicit implementations (Hindmarsh and Payne, 1996). It also reduces interprocess communication when implemented in parallel.

However, existing numerical models solve the time-dependent SIA using explicit or semi-implicit time-stepping (Hindmarsh and Payne, 1996; Huybrechts and others, 1996; Bueler and others, 2005; Egholm and Nielsen, 2010; Jarosch and others, 2013). Time-stepping restrictions are required in that context to avoid classical instabilities at wavelengths comparable with the grid spacing (Morton and Mayers, 2005). However, Bueler and others (2005) and Jarosch and others (2013) point out that these schemes also involve ad hoc treatment of the free margin of the ice sheet, for example using projection to reset computed negative thicknesses back to zero. It would be desirable to escape both time-stepping restrictions and model the margins in a mathematically principled manner. Both the goals are achievable if one implicitly solves the SIA as a free-boundary problem.

Early work on the free-boundary problem in one horizontal dimension (Hindmarsh and others, 1987) avoided ad hoc margin treatment by tracking it as a moving grid point. However, such margin-tracking does not easily extend to two dimensions (2D) because the margin of a real ice sheet is a curve of minimal smoothness and unknown-inadvance topology. Calvo and others $(2000,2002)$ describe the time-dependent free-boundary problem as a parabolic complementarity problem, including the constraint that ice thickness is never negative, but their work is limited to one horizontal dimension and flat bed. They also solve each time step numerically by a projected Gauss-Seidel scheme (Ciarlet, 2002), which does not scale to large problem sizes.

Jouvet and Bueler (2012) pose and numerically solve the steady-state free-boundary problem as a variational inequality (Kinderlehrer and Stampacchia, 1980) in two spatial dimensions and with non-trivial bed topography. Because this variational inequality is not equivalent to a minimization problem, in the general (non-flat-bed) case, they solve it by iterating well-posed but only approximate minimization problems that converge to the full equations in a fixed-point limit. The success of this method is demonstrated in a $5 \mathrm{~km}$ resolution steady-state calculation for Greenland using a piecewise-linear triangulation finite element (FE) method (Elman and others, 2005). In related work, Jouvet and Gräser (2013) solve the SIA component of their time-stepping hybrid ice dynamics model (Winkelmann and others, 2011) through a sequence of minimization problems that use the thickness from the previous time step. Each minimization problem is solved by a constraint-adapted nonlinear multigrid Newton iteration.

This paper follows Jouvet and Bueler (2012) by solving the steady-state free-boundary problem, but it uses a Mahaffylike scheme on a structured rectangular mesh, and applies the Newton iteration directly to the SIA equations. A continuation scheme generates an iterate within the domain of convergence of the Newton method, which then exhibits quadratic convergence. We formulate the discrete problem as a nonlinear complementarity problem (Benson and Munson, 2006) and solve it in parallel using either of two solvers from the open-source PETSc library (Balay and others, 2015). Because we have successfully solved the 
whole free-boundary problem all at once, our improved scheme is unconditionally stable as a fully implicit scheme, at least when the bed is not too rough.

The first part of this paper has a historical side. We reinterpret the classical Mahaffy scheme as using a non-standard quadrature choice in the conservation-of-mass flux integral. In this reinterpretation the approximate ice thickness lives in a continuous space of trial functions, unlike in the original FD scheme. These trial functions are piecewise-bilinear on a structured grid of rectangles, that is, they are $Q^{1} \mathrm{FEs}$ (Elman and others, 2005). Our reinterpretation has both finite volume (FV; LeVeque, 2002) and FE aspects. It is natural to call the scheme a 'finite volume element' method (FVE; Cai, 1990; Ewing and others, 2002) because the weak form is simply the flux integral itself. Adopting FVE thinking gives the best of both (i.e. FE and FV) worlds from the point of view of understanding the parts of the scheme.

This paper is organized as follows. Starting with a statement of the steady, isothermal SIA model, the classical Mahaffy scheme is recalled and then reinterpreted. Better quadrature in the flux integral, and a bit of first-order upwinding on the part of the flux that comes from the bedrock slope, are then added. The resulting improved scheme, which has the same stencil as the classical Mahaffy scheme, is called ' $M{ }^{\star \prime}$. The Newton solver for the discrete equations and constraints is then introduced. Initial numerical results in verification cases are excellent for a flat-bed dome exact solution and better than those from published higher-resolution upwind schemes on a bedrock-step exact solution. Then the method is applied to a real ice sheet by computing the steady-state shape of the Greenland ice sheet in a present-day modeled climate, at high resolution including sub-kilometer grids. An Appendix addresses the analytical calculation of the Jacobian in the $M^{\star}$ scheme.

\section{CONTINUUM MODEL}

The time-dependent evolution equation for the ice thickness $H$ is a statement of mass conservation:

$$
\frac{\partial H}{\partial t}+\nabla \cdot \mathbf{q}=m
$$

Here $\mathbf{q}$ denotes the vertically integrated flux and $m$ is the surface mass balance, also called the accumulation/ablation function. The SIA, the simplest relationship between $H$ and $\mathbf{q}$ in common use in glaciology, is a lubrication approximation (Fowler, 1997) of the Stokes equations for ice in non-sliding contact with the bed. We only consider the isothermal, Glen's flow law (Greve and Blatter, 2009) case. Let $b$ be the bed elevation and $s=H+b$ the ice surface elevation. The flux $\mathbf{q}$ is given by

$$
\mathbf{q}=-\Gamma H^{n+2}|\nabla s|^{n-1} \nabla s .
$$

Here $\Gamma=2 A(\rho g)^{n} /(n+2)$ is a positive constant derived from the power $n \geq 1$ and ice softness $A$ in Glen's flow law, and from the ice density $\rho$ and gravity $g$.

The flux $\mathbf{q}$ has several factored forms equivalent to Eqn (2). For instance, because it is common to combine Eqns (1) and (2) into a nonlinear diffusion equation (Huybrechts and others, 1996), one may write

$$
\mathbf{q}=-D \nabla s \quad \text { where } \quad D=\Gamma H^{n+2}|\nabla s|^{n-1} .
$$

Diffusion interpretation Eqn (3) is fully appropriate in the flatbed case because Eqns (1) and (2) can be transformed to a $p$-Laplacian diffusion equation (Calvo and others, 2002), but in general a diffusion interpretation is obscured by the bed gradient $\nabla b$. If the bed is not flat then $\nabla s$ and $\nabla H$ are different, so $\mathbf{q}$ is not strictly diffusive because it is not opposite to the gradient of the conserved quantity, namely $H$. For the same reason, non-flat beds are a barrier to proving wellposedness of the SIA (Jouvet and Bueler, 2012).

As an alternative to the diffusion form, one can compute a vertically averaged velocity $\mathbf{V}$, and then treat the flux as arising from the transport of $H$ by $\mathbf{V}$ :

$$
\mathbf{q}=\mathbf{V} H \quad \text { where } \quad \mathbf{V}=-\Gamma H^{n+1}|\nabla s|^{n-1} \nabla s .
$$

In this case, Eqn (1) is apparently a hyperbolic conservation equation, but this appearance is also deceiving because the velocity depends in part on the gradient of the transported quantity.

Furthermore, non-zero $\nabla b$ generates numerical conservation errors at the ice margin. In addressing such errors, Jarosch and others (2013) propose a third form for the flux, namely

$$
\mathbf{q}=\boldsymbol{\omega} H^{n+2}, \quad \text { where } \quad \boldsymbol{\omega}=-\Gamma|\nabla s|^{n-1} \nabla s .
$$

The vector field $\boldsymbol{\omega}$ can be thought of as a 'velocity' which transports $H^{n+2}$. In this thinking the combination of Eqns (1) and (5) becomes a kind of nonlinear hyperbolic equation, but again $\boldsymbol{\omega}$ depends on the gradient of the transported quantity $H$, so the combined equation is not truly hyperbolic.

We instead modify forms (3) and (5) to a split form

$$
\mathbf{q}=-D \nabla H+\mathbf{W} H^{n+2}, \quad \text { where } \quad \mathbf{W}=-\Gamma|\nabla s|^{n-1} \nabla b,
$$

and where $D$ is the same as in Eqn (3). The vector field $\mathbf{W}$ transports $H^{n+2}$, and is proportional to $\nabla b$. Only the magnitude of $\mathbf{W}$ is influenced by $\nabla H$. Note that $\mathbf{W}=0$ in the case of flat beds, while $\boldsymbol{\omega}$ is non-zero and $\mathbf{q}$ is actually diffusive in that case.

The combination of Eqn (1) with any of the above flux forms (2-6) defines a highly nonlinear diffusion-advection equation. It is important to note that Eqns (2-6) all describe exactly the same flux, even though the different appearances have often influenced modelers' choices of numerical scheme details. Form (6) has the numerical advantage that we can apply a non-oscillatory transport scheme to $\mathbf{W} H^{n+2}$ while also preserving accuracy by applying a centered scheme to $-D \nabla H$. Also, the often-dominant diffusion $-D \nabla H$ is a strong motivation to use implicit time-stepping, while implicit steps are not a common approach for hyperbolic problems.

In this paper, we will primarily solve the steady-state form of Eqn (1), namely

$$
\nabla \cdot \mathbf{q}=m
$$

By the divergence theorem, applied to any subregion $V$ of $\Omega$, we get the flux-integral form, equivalent to Eqn (7), namely

$$
\int_{\partial V} \mathbf{q} \cdot \mathbf{n} \mathrm{d} s=\int_{V} m \mathrm{~d} x \mathrm{~d} y .
$$

Here $\partial V$ denotes the boundary of $V, \mathbf{n}$ is the outward normal unit vector, and ds is the length element on the 
closed curve $\partial V$. Our numerical schemes will be based on Eqn (8).

Equation (8) is solved as a boundary-value problem where both $H=0$ and $\mathbf{q}=0$ apply along the (free) boundary. However, the location where those boundary conditions apply is unknown (Jouvet and Bueler, 2012; Jarosch and others, 2013). The ice-covered domain where Eqn (8) applies cannot be treated as a small modification of a known domain, though that approach can be taken in explicit time-stepping schemes for Eqn (1) (Huybrechts and others, 1996; Bueler and others, 2005).

To be more precise about the free-boundary conditions, the input data consist only of the bed elevation $b(x, y)$ and the (steady) surface mass balance $m(x, y)$. These input data must be defined on a larger, fixed computational domain $\Omega$ than the ice-covered set. If the surface mass balance $m$ is sufficiently negative in the region near the boundary of $\Omega$ on which Eqn (8) is solved, then $H \rightarrow 0$ at locations inside $\Omega$, namely at the ice margin (free boundary). But also $\mathbf{q} \rightarrow 0$ at the same locations because ice does not flow past the margin. Because of the factor $H^{n+2}$ in Eqn (3) for the diffusivity $D$, the problem has degenerate diffusivity, that is, $D \rightarrow 0$ at the free boundary. Solving Eqn (8), or computing a time step of Eqn (1), as such a free-boundary problem, without a boundary flux applied along any part of the (fixed) boundary of $\Omega$, is usually called a 'whole' ice-sheet model. We restrict our attention to such whole ice-sheet models.

In this paper, we solve coupled Eqns (6) and (8), with $D$ computed as in Eqn (3). As noted above, we assume $m$ is sufficiently negative in the region near the boundary of $\Omega$, so that the ice-covered set is surrounded by ice-free areas. Any contact with the ocean must be modeled as strongly negative values of $m$, and there is no modeled floating ice. The solution is the non-negative thickness function $H(x, y)$, defined everywhere in $\Omega$ and equal to zero where there is no ice.

\section{METHODS}

\subsection{Classical Mahaffy scheme}

Consider the rectangular structured FD grid, with spacing $\Delta x$, $\Delta y$, shown in Figure 1a. The Mahaffy (1976) scheme, which is also 'method 2' in Hindmarsh and Payne (1996), calculates a flux component at each of the staggered grid points based on values at regular points $\left(x_{j}, y_{k}\right)$. For the staggered grid we introduce notation:

$$
x_{j}^{ \pm}=x_{j} \pm \frac{\Delta x}{2}, \quad y_{k}^{ \pm}=y_{k} \pm \frac{\Delta y}{2} .
$$

At $\left(x_{j}^{+}, y_{k}\right)$ the scheme computes the $x$-component of the flux by

$$
q_{j+(1 / 2), k}^{x}=-\Gamma\left(\hat{H}_{j+(1 / 2), k}\right)^{n+2}\left(\alpha_{\triangleright}\right)^{n-1} \frac{s_{j+1, k}-s_{j, k}}{\Delta x},
$$

where $s_{j, k}=H_{j, k}+b_{j, k}$

$$
\hat{H}_{j+(1 / 2), k}=\frac{H_{j, k}+H_{j+1, k}}{2},
$$

and ' $\alpha$ ' is an estimate of the slope $|\nabla s|$ at $\left(x_{j}^{+}, y_{k}\right)$ given by

$$
\begin{aligned}
\left(\alpha_{\triangleright}\right)^{2}= & \left(\frac{s_{j+1, k}-s_{j, k}}{\Delta x}\right)^{2} \\
& +\left(\frac{s_{j, k+1}+s_{j+1, k+1}-s_{j, k-1}-s_{j+1, k-1}}{4 \Delta y}\right)^{2} .
\end{aligned}
$$

At $\left(x_{j}, y_{k}^{+}\right)$the scheme computes

$$
q_{j, k+(1 / 2)}^{y}=-\Gamma\left(\hat{H}_{j, k+(1 / 2)}\right)^{n+2}\left(\alpha_{\bullet}\right)^{n-1} \frac{s_{j, k+1}-s_{j, k}}{\Delta y},
$$

where $\hat{H}_{j, k+(1 / 2)}$ and $\alpha$ are defined by swapping the roles of $j$ and $k$, and $\Delta x$ and $\Delta y$, in Eqns (11) and (12). The slope approximation in Eqn (12) is perhaps the least-obvious aspect of the Mahaffy scheme, but one may check that these FD formulas are consistent (Morton and Mayers, 2005) with Eqn (2).

The discretization of mass conservation, Eqn (7), uses straightforward centered differences (Morton and Mayers, 2005):

$$
\frac{q_{j+1 / 2, k}^{x}-q_{j-1 / 2, k}^{X}}{\Delta x}+\frac{q_{j, k+1 / 2}^{y}-q_{j, k-1 / 2}^{y}}{\Delta y}=m_{j, k},
$$

where $m_{j, k}=m\left(x_{j}, y_{k}\right)$. Equation (14) relates the nine unknown values of $H$ at the regular grid points in Figure 1a, thus giving the 'stencil' of the scheme. It is required to hold at all regular grid points, thus forming a (generally) large, but finite, non-linear algebraic system.

\subsection{FVE reinterpretation}

The above description of the Mahaffy FD method is familiar to numerical ice-sheet modelers, but we now re-derive the scheme from an FVE perspective. Our reinterpretation uses the same structured grid, but the regular grid points are now nodes (degrees of freedom) for a continuous space of trial functions. Any FE method supposes that an approximation $H^{h}$ of the solution lies in some finite-dimensional space of functions that are sufficiently well-behaved so that the approximate flux $\mathbf{q}^{h}$ is defined almost everywhere. In an FV method, however, an integral equation like Eqn (8) is required to hold for a finite set of control volumes $V$ which tile $\Omega$ (LeVeque, 2002).

In Figure $1 b$, element $\square_{j, k}$ is the rectangle with lower-left corner at $\left(x_{j}, y_{k}\right)$. When associated with bilinear functions this rectangle is a $Q^{1} \mathrm{FE}$ (Elman and others, 2005). A basis for bilinear functions on $\square_{j, k}$ is the set

$$
\chi_{\ell}\left(\frac{x-x_{j}}{\Delta x}, \frac{y-y_{k}}{\Delta y}\right)
$$

for $\ell=0,1,2,3$, where

$$
\begin{aligned}
& \chi_{0}(\xi, \eta)=(1-\xi)(1-\eta), \quad \chi_{1}(\xi, \eta)=\xi(1-\eta) \\
& \chi_{2}(\xi, \eta)=\xi \eta, \quad \chi_{3}(\xi, \eta)=(1-\xi) \eta
\end{aligned}
$$

With this order, $\chi_{\ell}=1$ on element corners traversed in counterclockwise order (Fig. 1b).

Let $S_{h}$ be the trial space of functions that are continuous on the whole computational domain $\Omega$ and bilinear on each element $\square_{j, k}$. Functions in $S_{h}$ have a bounded gradient that is defined almost everywhere, but the gradient is discontinuous along the element edges (solid lines in Fig. 1b). We write $\psi_{j, k}$ 

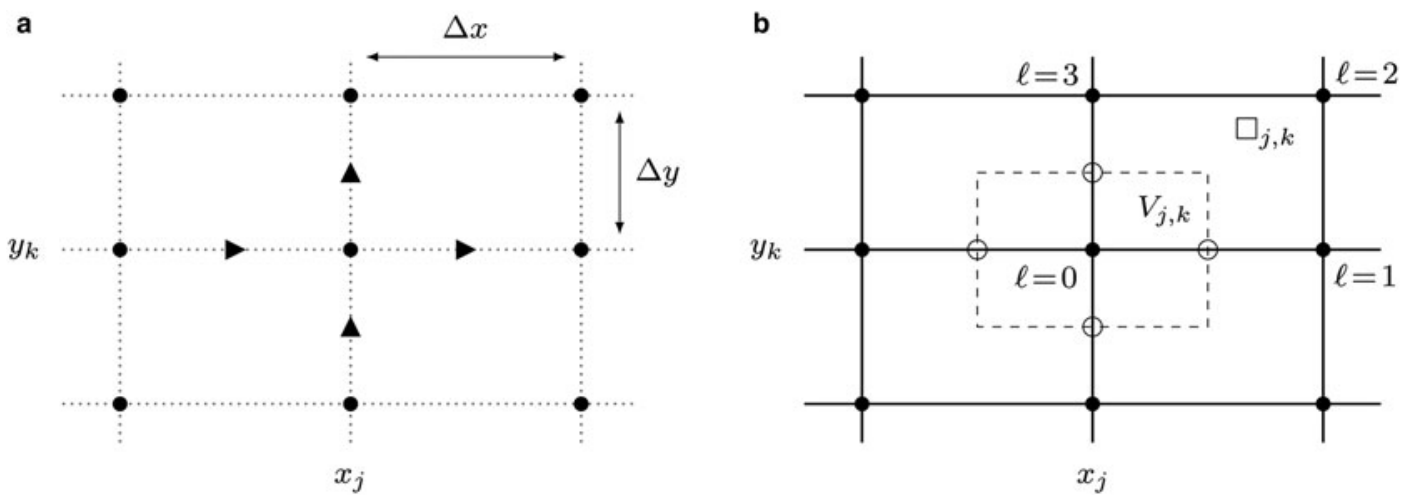

Fig. 1. (a) A structured FD grid with regular (dots) and staggered (triangles) points. (b) The same grid as an FVE grid with rectangular elements $\square_{j, k}$ (solid), nodes (dots), a dual rectangular control volume $V_{j, k}$ (dashed) and Mahaffy's flux-evaluation locations (circles). The corners of element $\square_{j, k}$ are locally indexed by $\ell$.

$(x, y)$ for the unique function in $S_{h}$ so that $\psi_{j, k}\left(x_{r}, y_{s}\right)=\delta_{j r} \delta_{k s}$ (Fig. 2a); such functions form a basis of $S_{h}$. We seek an approximate solution $H^{h}$ from $S_{h}$. Also let $b^{h}$ in $S_{h}$ be the interpolant of the bed elevation data $b$, and let $s^{h}=H^{h}+b^{h}$. We denote by $\mathbf{q}^{h}$ the flux computed from formula (6) using $H^{h}, b^{h}$ and $s^{h}$, so that $\mathbf{q}^{h}$ is well defined on the interior of each element.

Let $V_{j, k}$ be the control volume with center at $\left(x_{j}, y_{k}\right)$ shown in Figure 1b. In the FVE method, we require Eqn (8) to hold for $\mathbf{q}=\mathbf{q}^{h}$ and $V$ equal to each $V_{j, k}$. Because we use a periodic grid with $N_{x}$ rectangles in the $x$-direction and $N_{y}$ in the $y$-direction, there are $N=N_{x} N_{y}$ nodes, $N$ distinct control volumes and $N$ equations in the algebraic system.

Instead of control volumes, in a full FE interpretation we would introduce test functions. We can also do this for our scheme, as follows. Let $S_{h}^{*}$ be the space of functions that are constant on each control volume $V_{j, k}$. These functions are piecewise-constant and discontinuous along the control volume edges. A basis of $S_{h}^{*}$ is formed by those functions which are one at a single node and zero at all other nodes; Figure $2 b$ shows such a function $\omega_{j, k}$. Requiring Eqn (8) to hold for each control volume in our FVE method is equivalent to multiplying Eqn (7) by $\omega_{j, k}$ and then integrating by parts. Showing the equivalence would require generalized functions, however, as the derivative of a step function is a Dirac delta function. Because we adopt the FVE interpretation, we have no further need for test functions, the space $S_{h}^{*}$ or generalized functions.

Both this reinterpreted Mahaffy scheme, and our improved scheme below, assume midpoint quadrature on the right-hand integral in Eqn (8). Thus, we seek $H^{h}$ in $S_{h}$ satisfying

$$
\int_{\partial V_{j, k}} \mathbf{q}^{h} \cdot \mathbf{n} \mathrm{d} s=m_{j, k} \Delta x \Delta y
$$

for all $j, k$.

However, it remains to do quadrature on the left in Eqn (16). We decompose the integral over the four edges of $\partial V_{j, k}$ :

$$
\begin{aligned}
\int_{\partial V_{j, k}} \mathbf{q}^{h} \cdot \mathbf{n} \mathrm{d} s= & \int_{y_{k}^{-}}^{y_{k}^{+}} q^{x}\left(x_{j}^{+}, y\right) \mathrm{d} y \\
& +\int_{x_{j}^{-}}^{x_{j}^{+}} q^{y}\left(x, y_{k}^{+}\right) \mathrm{d} x \\
& -\int_{y_{k}^{-}}^{y_{k}^{+}} q^{x}\left(x_{j}^{-}, y\right) \mathrm{d} y \\
& -\int_{x_{j}^{-}}^{x_{j}^{+}} q^{y}\left(x, y_{k}^{-}\right) \mathrm{d} x .
\end{aligned}
$$

The flux $\mathbf{q}^{h}$ is a bounded function, but it is discontinuous across element boundaries. In the first right-hand integral in Eqn (17), the integrand has a jump discontinuity at the midpoint of the interval of integration (i.e. at $y=y_{k} ;$ note $\partial s^{h} / \partial y=s_{y}^{h}$ is discontinuous there), but formula (10) in the Mahaffy FD scheme computes the normal component of $\mathbf{q}^{h}$ exactly at that midpoint.

The key to reinterpreting the Mahaffy scheme is that it computes each integral in Eqn (17) by the midpoint method using averages of the discontinuous surface gradient across its jump discontinuities. The scheme does not use true
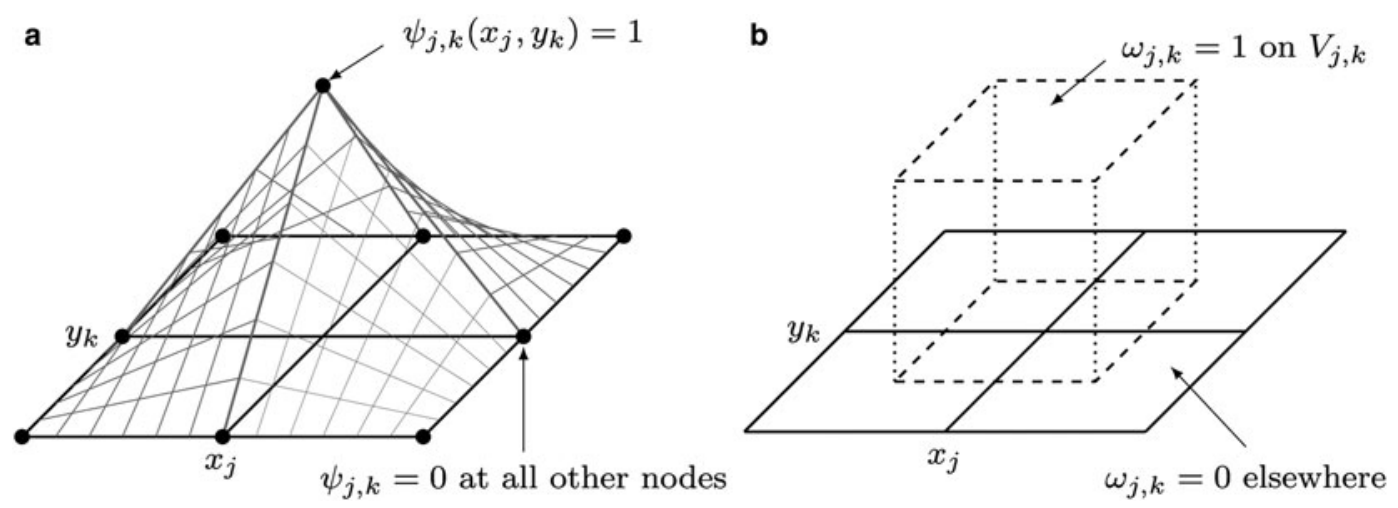

Fig. 2. (a) A continuous 'hat' basis function $\psi_{j, k}$ in the trial space $S_{h}$. (b) A full FE interpretation of our scheme would use piecewise-constant basis functions $\omega_{j, k}$ from the test space $S_{h}^{*}$. 
quadrature because the integrand $\mathbf{q}^{h} \cdot \mathbf{n}$ does not have a value at the quadrature point. To turn this idea into formulas, first observe that both the thickness $H^{h}$ and the $x$-derivative $\partial s^{h} / \partial x=s_{x}^{h}$ are continuous along the edge between elements $\square_{j, k}$ and $\square_{j, k-1}$. In fact, using element basis (15), the surface gradient on $\square_{j, k}$ has components

$$
\begin{aligned}
s_{x}^{h}(x, y)= & \frac{s_{j+1, k}-s_{j, k}}{\Delta x}\left(1-\frac{y-y_{k}}{\Delta y}\right) \\
& +\frac{s_{j+1, k+1}-s_{j, k+1}}{\Delta x}\left(\frac{y-y_{k}}{\Delta y}\right), \\
s_{y}^{h}(x, y)= & \frac{s_{j, k+1}-s_{j, k}}{\Delta y}\left(1-\frac{x-x_{j}}{\Delta x}\right) \\
& +\frac{s_{j+1, k+1}-s_{j+1, k}}{\Delta y}\left(\frac{x-x_{j}}{\Delta x}\right) .
\end{aligned}
$$

On $\square_{j, k-1}, s_{x}^{h}$ and $s_{y}^{h}$ can be calculated by shifting the index $k$ to $k-1$. Thus, the continuous function $s_{x}^{h}$ has value

$$
s_{x}^{h}\left(x_{j}^{+}, y_{k}\right)=\frac{s_{j+1, k}-s_{j, k}}{\Delta x}
$$

at the midpoint in the first integral in Eqn (17). Similarly, writing out $H^{h}$ using element basis (15) gives

$$
H^{h}\left(x_{j}^{+}, y_{k}\right)=\frac{H_{j, k}+H_{j+1, k}}{2}
$$

at the midpoint, which is Eqn (11). The $y$-derivative $s_{y^{\prime}}^{h}$ however, has different values above (on $\square_{j, k}$ ) and below (on $\square_{j, k-1}$ ) the element boundary at $y=y_{k}$. The limits are:

$$
\begin{aligned}
& \lim _{y \rightarrow y_{k}^{+}} s_{y}^{h}\left(x_{j}^{+}, y\right)=\frac{s_{j, k+1}-s_{j, k}+s_{j+1, k+1}-s_{j+1, k}}{2 \Delta y}, \\
& \lim _{y \rightarrow y_{k}^{-}} s_{y}^{h}\left(x_{j}^{+}, y\right)=\frac{s_{j, k}-s_{j, k-1}+s_{j+1, k}-s_{j+1, k-1}}{2 \Delta y} .
\end{aligned}
$$

The average of these values is not a value of $s_{y}^{h}$, but a reconstruction:

$$
\widehat{s_{y}^{h}}\left(x_{j}^{+}, y_{k}\right)=\frac{s_{j, k+1}+s_{j+1, k+1}-s_{j, k-1}-s_{j+1, k-1}}{4 \Delta y} .
$$

Formula (22) is exactly the estimate of $\partial s / \partial y$ which appears in FD formula (12).

In our FVE reinterpretation, the Mahaffy FD scheme uses Eqns (19), (20) and (22) in the midpoint rule for the first integral in Eqn (17):

$$
\begin{aligned}
& \int_{y_{k}^{-}}^{y_{k}^{+}} q^{x}\left(x_{j}^{+}, y\right) d y \\
& \quad \approx-\Delta y \Gamma\left(H^{h}\left(x_{j}^{+}, y_{k}\right)\right)^{n+2}(\alpha)^{n-1} s_{x}^{h}\left(x_{j}^{+}, y_{k}\right),
\end{aligned}
$$

where

$$
\left(\alpha_{\bullet}\right)^{2}=s_{x}^{h}\left(x_{j}^{+}, y_{k}\right)^{2}+\widehat{s_{y}^{h}}\left(x_{j}^{+}, y_{k}\right)^{2}
$$

is the same as in Eqn (12). Thus, the FD scheme approximates each integral on the right in Eqn (17) by a perfectly reasonable quadrature 'crime' (cf. Strang, 1972) which averages across a discontinuity to reconstruct a slope. Recognizing the Mahaffy choice as quadrature-like, in this FVE context, is beneficial because it allows us to improve the scheme.

\subsection{Improved quadrature}

If the goal is to accurately generate an algebraic equation from Eqn (16) by quadrature along $\partial V_{j, k}$, then it is easy to improve the quadrature. We also use flux decomposition Eqn (6) with an upwind-type discretization on the bed gradient term $\mathbf{W} H^{n+2}$ (Section 3.4). Together these improvements define our new ' $M{ }^{\star \prime}$ scheme.

As already noted, the numerical approximation $\mathbf{q}^{h}$ from Eqn (6) is defined and smooth on the interior of each element, but discontinuous across element boundaries. So we break each interval of integration on the right-hand side of Eqn (17) into two parts and use the midpoint rule, the optimal one-point rule, on each half. For example, we break the first integral at $y=y_{k}$ :

$$
\begin{aligned}
& \int_{y_{k}^{-}}^{y_{k}^{+}} q^{x}\left(x_{j}^{+}, y\right) d y \\
& \quad=\int_{y_{k}^{-}}^{y_{k}} q^{x}\left(x_{j}^{+}, y\right) d y+\int_{y_{k}}^{y_{k}^{+}} q^{x}\left(x_{j}^{+}, y\right) d y \\
& =\frac{\Delta y}{2}\left(q^{x}\left(x_{j}^{+}, y_{k}-\frac{\Delta y}{4}\right)+q^{x}\left(x_{j}^{+}, y_{k}+\frac{\Delta y}{4}\right)\right) .
\end{aligned}
$$

Recalling notation (9), values $q^{x}\left(x_{j}^{+}, y_{k} \pm(\Delta y / 4)\right)$ are evaluations of $\mathbf{q}^{h}$ at points of continuity.

Similar formulas apply to the other three integrals on the right-hand side of Eqn (17). Figure 3 shows all eight quadrature points needed to compute the full integral over $\partial V_{j, k}$ in Eqn (16). At each point we evaluate the $x$ - or $y$-component of $\mathbf{q}^{h}$ and multiply by a constant to get the appropriate integral. Thus, our approximation of Eqn (16) is

$$
\sum_{s=0}^{7} \mathbf{c}_{s} \cdot \mathbf{q}^{h}\left(x_{j}^{s}, y_{k}^{s}\right)=m_{j, k} \Delta x \Delta y,
$$

where

$$
\begin{aligned}
& \mathbf{c}_{0}=\mathbf{c}_{7}=\left(0, \frac{\Delta y}{2}\right), \\
& \mathbf{c}_{1}=\mathbf{c}_{2}=\left(\frac{\Delta x}{2}, 0\right), \\
& \mathbf{c}_{3}=\mathbf{c}_{4}=\left(0,-\frac{\Delta y}{2}\right), \\
& \mathbf{c}_{5}=\mathbf{c}_{6}=\left(-\frac{\Delta x}{2}, 0\right),
\end{aligned}
$$

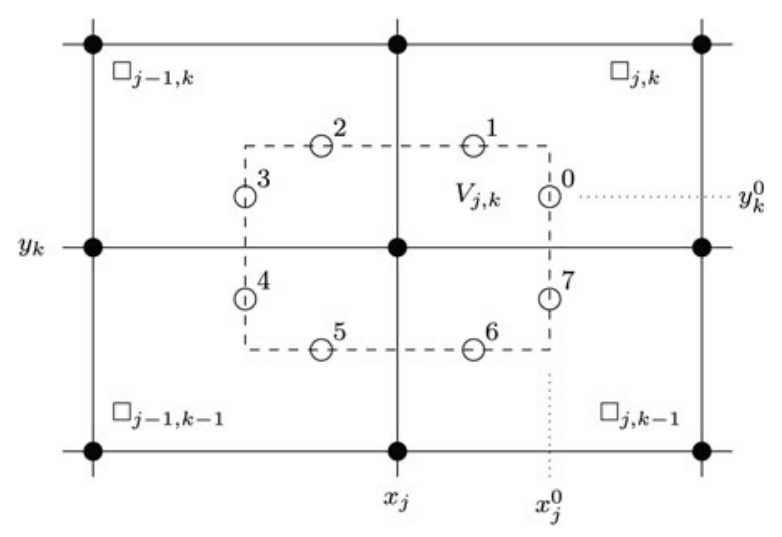

Fig. 3. For Eqn (26) we evaluate $\mathbf{q}^{h}(x, y)$ at eight quadrature points (numbered circles) along $\partial V_{j, k}$ (dashed). 
and

$$
\begin{aligned}
& x_{j}^{0}=x_{j}^{7}=x_{j}+\frac{\Delta x}{2}, \quad y_{k}^{1}=y_{k}^{2}=y_{k}+\frac{\Delta y}{2}, \\
& x_{j}^{1}=x_{j}^{6}=x_{j}+\frac{\Delta x}{4}, \quad y_{k}^{0}=y_{k}^{3}=y_{k}+\frac{\Delta y}{4}, \\
& x_{j}^{2}=x_{j}^{5}=x_{j}-\frac{\Delta x}{4}, \quad y_{k}^{4}=y_{k}^{7}=y_{k}-\frac{\Delta y}{4}, \\
& x_{j}^{3}=x_{j}^{4}=x_{j}-\frac{\Delta x}{2}, \quad y_{k}^{5}=y_{k}^{6}=y_{k}-\frac{\Delta y}{2} .
\end{aligned}
$$

Implementing Eqn (26) therefore requires evaluating $\mathbf{q}^{h}$ at eight quadrature points, twice the number for the classical method, but the stencils are the same because we use the same nine nodal values of $H^{h}$.

One could propose further-improved quadrature, replacing the midpoint rule by higher-order methods like two-point Gauss-Legendre. Also, our $Q^{1}$ elements could be replaced by higher-order (e.g. $Q^{2}$ ) elements. Though such methods have not been tested, in fact the largest numerical SIA errors occur near the ice margin where the solution $H$ has unbounded gradient (Bueler and others, 2005). Thus, higher-order quadrature and interpolation will not give much advantage. We believe our improved method represents a measurable accuracy and Newton-iteration-convergence improvement over the classical Mahaffy method (Section 4) because it evaluates $\mathbf{q}^{h}$ at points of continuity, not because the order of quadrature or interpolation is flawed in the classical scheme.

\subsection{Improvement from upwinding}

Jarosch and others (2013) show that the Mahaffy scheme can suffer from significant mass-conservation errors at locations of abrupt change in the bed elevation. In such cases where the bed gradient dominates the flux, the mass-conservation equation has more 'hyperbolic' character. Thus, Jarosch and others use a high-resolution upwind scheme (LeVeque, 2002) based on flux form Eqn (5). They show reduced mass-conservation errors in the sense of giving numerical solutions that are closer in volume to an exact solution. On the other hand, their scheme, which solves the time-dependent Eqn (1) using explicit time-stepping, expands the stencil because it needs values two grid spaces away.

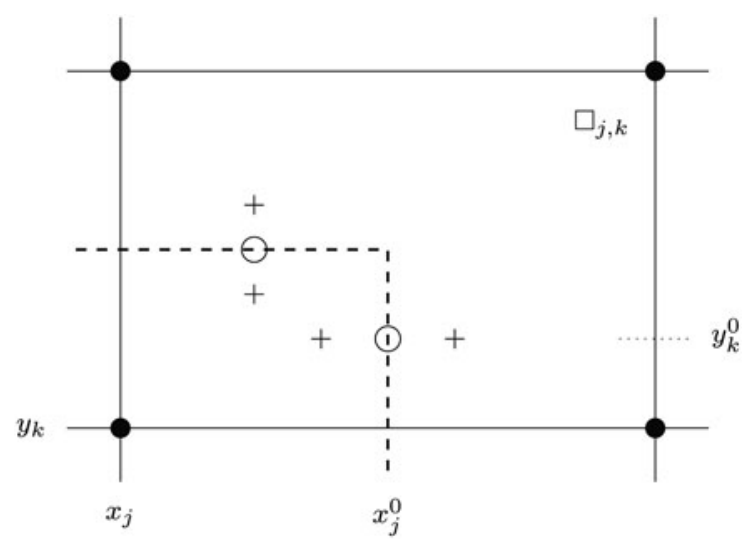

Fig. 4. On element $\square_{j, k}$, when computing the flux at quadrature points (circles), upwinding of the flux term $\tilde{\mathbf{q}}=\mathbf{W} H^{n+2}$ evaluates the thicknesses $H$ at locations shown with ' + ', one-quarter of the way to the element boundary.
By comparison, we propose using minimal first-order upwinding based on form (6) of the flux, even though upwinding is not required for either linear stability or nonoscillation of our implicit scheme (Morton and Mayers, 2005). Numerical testing suggests upwinding is effective in our case because it improves the conditioning of the Jacobian matrix used in the Newton iteration (not shown).

In our improved scheme, the transport-type flux $\tilde{\mathbf{q}}=$ $\mathbf{W} H^{n+2}$ uses $H$ evaluated at a location different from the quadrature point, according to the direction of $\mathbf{W}$ evaluated at the quadrature point. For instance, our upwind modification at $\left(x_{j}^{0}, y_{k}^{0}\right)$ - see Figure 3 - uses the sign of

$$
W_{*}^{x}=W^{x}\left(x_{j}^{0}, y_{k}^{0}\right)
$$

where $\mathbf{W}=\left(W^{x}, W^{y}\right)$. Note that the sign of $W_{*}^{x}$ is opposite that of the $x$-component of $\nabla b^{h}$ at $\left(x_{j}^{0}, y_{k}^{0}\right)$. We shift the evaluation of $H$ upwind by a fraction $0 \leq \lambda \leq 1$ of an element width,

$$
\begin{array}{r}
\tilde{\mathbf{q}}^{x}\left(x_{j}^{0}, y_{k}^{0}\right) \\
\quad=W_{*}^{x} \begin{cases}H\left(x_{j}^{0}-\lambda \frac{\Delta x}{2}, y_{k}^{0}\right)^{n+2}, & W_{*}^{x} \geq 0, \\
H\left(x_{j}^{0}+\lambda \frac{\Delta x}{2}, y_{k}^{0}\right)^{n+2}, & W_{*}^{x}<0,\end{cases}
\end{array}
$$

where $\lambda=0$ is no upwinding and $\lambda=1$ is the maximum upwinding that does not expand the stencil. After experimentation (Section 4) we have chosen $\lambda=1 / 4$ (Fig. 4). This value is seen to be large enough to improve the convergence of the Newton iteration but also small enough to generate substantial improvements in accuracy for the bedrock-step exact solution. Upwinding at the other seven points along $\partial V_{j, k}$ (Fig. 3) uses similar formulas.

The ' $M^{\star \prime}$ scheme combines both of the above improvements. We will see in verification (Section 4) that it achieves higher solution accuracy than either the apparently secondorder Mahaffy scheme or the higher-resolution explicit advection scheme of Jarosch and others (2013). (Note that the additional non-smoothness of such high-resolution flux-limiting methods suggests caution when using a Newton scheme, which needs a differentiable residual function.) Convergence of the Newton iteration is also improved compared with the classical Mahaffy scheme. Evidence from both verification and realistic (Section 4) cases suggests that our form of upwinding is most important at locations of low regularity of the solution.

\subsection{Solution of the equations}

It remains to describe the numerical solution of the system of highly nonlinear algebraic equations that is generated by the formulas above. This is additionally non-trivial because an inequality constraint applies to each unknown.

Each equation from the $M^{*}$ scheme is Eqn (26) with an upwind modification like (30) at the quadrature points. The resulting system of $N=N_{x} N_{y}$ equations is

$$
F_{j, k}(\mathbf{H})=0 .
$$

This determines $N$ unknowns, a vector $\mathbf{H}=\left\{H_{j, k}\right\}$; note $H^{h}(x, y)$ and $\mathbf{H}$ are actually two representations of the same discrete thickness approximation. System (31) is not adequate by itself, however, because each unknown thickness $H_{j, k}$ must be non-negative, that is, $\mathbf{H} \geq 0$. 
These requirements can be combined into a variational inequality (Kinderlehrer and Stampacchia, 1980; Jouvet and Bueler, 2012). Equivalently, we can write them in nonlinear complementarity problem form (Benson and Munson, 2006):

$$
\mathbf{H} \geq 0, \quad \mathbf{F}(\mathbf{H}) \geq 0, \quad \mathbf{H} \cdot \mathbf{F}(\mathbf{H})=0 .
$$

In fact, we will solve (32) by a 'constrained' Newton solver, specifically either by the reduced-space (RS) or semi-smooth (SS) methods (Benson and Munson, 2006) implemented in PETSc (Balay and others, 2015). Our open-source C code contains the residual and Jacobian evaluation subroutines. (To get the code and examples, clone the repository at https: // github.com/bueler/sia-fve. Then see README.md in directory petsc/.) However, the parallel grid management, Newton solver, iterative linear solver and linear preconditioning methods are all inside the PETSc library, and they are chosen at runtime. Both multigrid (Briggs and others, 2000) and additive-Schwarz-type domain decomposition (Smith and others, 1996) preconditioning are found to work (not shown), but the latter is more robust and is used in all runs in Section 4.

The Jacobian of system (31), that is, the $N \times N$ matrix,

$$
J=\left(\frac{\partial F_{j, k}}{\partial H_{p, q}}\right)
$$

can be computed via hand-calculated derivatives. This additional effort (Appendix) gives a speed-up only by a factor of $\sim 1.5$ over the alternative, namely FD computation of Jacobian entries coming from additional residual (i.e. F) evaluations. Such approximate Jacobians are efficiently implemented in PETSc using 'coloring' of the nodes (Curtis and others, 1974) so that, because of the nine-point stencil, only a total of ten residual evaluations are needed to approximate $J$.

In the standard theory of Newton's method, quadratic convergence should occur if $J$ is Lipschitz-continuous as a function of the unknowns (Kelley, 2003). However, the flux $\mathbf{q}$ here is generally not that smooth as a function of $\nabla H$. In particular, for $1<n<3$ the flux has a second derivative which is not Lipschitz-continuous with respect to changes in $\nabla H$. Because Eqn (26) already involves differentiating $\mathbf{q}$, in the limit where the control volume shrinks to zero, the behavior of second derivatives of $\mathbf{q}$ determines the smoothness of $J$ and thus the convergence of the solver. Non-smoothness is best seen in 1-D where Eqn (2) is

$$
q\left(H, H^{\prime}\right)=-\Gamma H^{n+2}\left|H^{\prime}+b^{\prime}\right|^{n-1}\left(H^{\prime}+b^{\prime}\right) .
$$

when $n>1$,

$$
\frac{\partial^{2} q}{\partial H^{\prime 2}}=-C H^{n+2}\left|H^{\prime}+b^{\prime}\right|^{n-3}\left(H^{\prime}+b^{\prime}\right)
$$

for $C>0$. If $n=2$, for example, this function undergoes a step at $H^{\prime}=-b^{\prime}$, and thus is not Lipschitz. In fact, Eqn (35) is not Lipschitz-continuous for $1<n<3$.

Because we want methods that work for all exponents $n \geq$ 1 , we regularize by replacing

$$
|\nabla s| \rightarrow\left(|\nabla s|^{2}+\delta^{2}\right)^{1 / 2}
$$

with $\delta=10^{-4}$, in computing $\mathbf{q}$ and its derivatives. This regularization, which makes little difference when the surface slope is of order $10^{-3}$ or larger, is similar to that used in regularizing the viscosity in the Stokes equations (Greve and Blatter, 2009) and other stress balance models (e.g. Bueler and Brown, 2009; Brown and others, 2013).

A Newton solver requires an initial iterate, and we generate it in two stages. First we apply a heuristic that seems to work adequately for both ice sheet- and glacierscale problems, and is used by Jouvet and Gräser (2013) in a time-stepping context. From the mass-balance data $m_{j, k}$ $\left(\mathrm{m} \mathrm{a}^{-1}\right)$ we compute

$$
H_{j, k}^{(0)}=\max \left\{0,1000 m_{j, k}\right\}
$$

which builds the initial thickness simply by piling up 1000 years of accumulation.

In the second stage, we apply a simple 'parameter continuation' scheme, to aid in the convergence of the Newton iteration on our degenerate, non-linear free-boundary problem. We first apply the Newton method to an easier free-boundary problem, one with constant diffusivity. Then we adjust a continuation parameter $\varepsilon$ until we solve the full SIA free-boundary problem. Specifically, for $0 \leq$ $\varepsilon \leq 1$ we define

$$
\begin{gathered}
n(\varepsilon)=(1-\varepsilon) n+\varepsilon n_{0} \quad \text { and } \\
D(\varepsilon)=(1-\varepsilon) D+\varepsilon D_{0},
\end{gathered}
$$

where $n$ is the original exponent, $D$ is computed in Eqn (3), $n_{0}=1$, and $D_{0}$ is a typical scale of diffusivities for the problem (e.g. $D_{0}=0.01 \mathrm{~m}^{2} \mathrm{~s}^{-1}$ for a glacier problem or $D_{0}=10 \mathrm{~m}^{2} \mathrm{~s}^{-1}$ for an ice sheet). Note $n(1)=n_{0}$ and $D(1)=D_{0}$, while $n(0)=n$ and $D(0)=D$. We consecutively solve free-boundary problems corresponding to 13 values of $\varepsilon$ :

$$
\varepsilon_{i}=\left\{\begin{array}{cc}
(0.1)^{i / 3}, & \text { for } i=0, \ldots, 11, \\
0, & i=12 .
\end{array}\right.
$$

At the last stage $\varepsilon_{12}=0$, the problem is the unmodified SIA. Note the parameter $\varepsilon_{i}$ is reduced by an order of magnitude as $i$ increases by 3 .

We start with $\varepsilon_{0}=1$, so we are solving (32) with values $n=n_{0}$ and $D=D_{0}$ and initial iterate $H^{(0)}$ from (37). Once this first stage converges, the ice margin has moved far from the equilibrium line - which is the margin of initial iterate (37) - into the ablation zone as expected. The solution is then used as the initial iterate for problem (32) with the second value $\varepsilon_{1}=(0.1)^{1 / 3} \approx 0.46$ in Eqn (38). Continuing in this way we eventually solve the unregularized $n(0)=n$ and $D(0)=D$ problem .

\subsection{Time-stepping}

The relationship between our steady-state computations and fully implicit time-stepping methods for Eqn (1) deserves examination. We will be able to exploit such time steps to make the Newton solver for the steady-state problem more robust for rough-bed cases.

The backward-Euler (Morton and Mayers, 2005) approximation of Eqn (1) is

$$
\frac{H^{\ell}-H^{\ell-1}}{\Delta t}+\nabla \cdot \mathbf{q}^{\ell}=m
$$


where $H^{\ell}(x, y) \approx H\left(t_{\ell}, x, y\right)$ is the unknown thickness, $H^{\ell-1}$ is known from the previous time step, and $\mathbf{q}^{\ell}$ is the flux computed using $H^{\ell}$. Our methods extend easily from solving Eqn (32) to solving Eqn (40), subject, as before, to the constraint $H^{\ell} \geq 0$.

Solving for steady state effectively requires taking infiniteduration implicit time steps in Eqn (40). Using finite $\Delta t$ is easier than the steady-state problem, however, both because the initial iterate can be taken to be the solution at the previous time step, and because the continuation sequence can either be avoided entirely or truncated to only include small $\varepsilon$ values. Even more important is the key fact that solving Eqn (40) gets easier as $\Delta t \rightarrow 0$, because the Jacobian / has a dominanting diagonal contribution from the $H^{\ell} / \Delta t$ term.

If a Newton iteration fails to converge in the steady-state case, we might hope that it would still converge for Eqn (40) using finite $\Delta t$, and this is what we see in practice. In fact, switching from the steady-state problem to long (e.g. 0.1-100 years depending on resolution) backward-Euler time steps is a 'recovery strategy' to cope with Newton solver difficulties when dealing with highly irregular bed topography data in the steady-state problem (Section 4).

\section{RESULTS}

We first apply our $M^{*}$ method in two examples where exact solutions are known. We use the exact solutions to evaluate the convergence of the discrete solution to the continuum solution under grid refinement (verification), and we evaluate the convergence of the continuation scheme and Newton iteration in these cases. After that we apply the method to highresolution Greenland ice-sheet bedrock topography. All computations in this section use physical parameters from European ice-sheet Modelling Initiative I (EISMINT I) (Huybrechts and others, 1996) and are in two horizontal variables, though we show some results along flowlines for clarity.

\subsection{Verification cases}

An angularly symmetric steady-state exact solution exists in the flat-bed case (Bueler, 2003; van der Veen, 2013). This 'dome' exact solution, with parameters suitable for a medium-sized ice sheet, is shown in Figure 5. The numerical

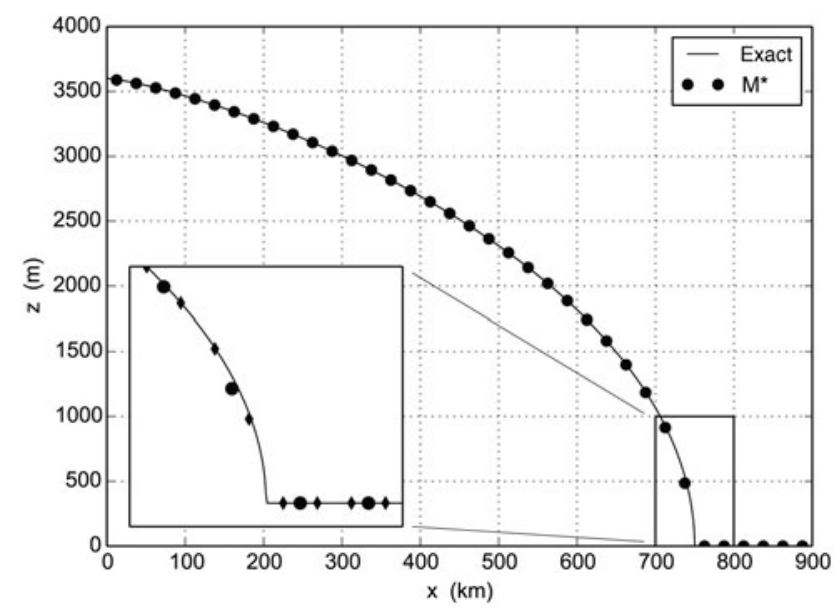

Fig. 5. Result from $M^{\star}$ method on a $\Delta x=\Delta y=25 \mathrm{~km}$ grid (dots) compared with the dome exact solution. The detail of the margin adds results from a $12.5 \mathrm{~km}$ grid (diamonds). results from our $M^{\star}$ method are very close to the exact solution, with numerical error at the last positive-thickness grid point barely visible.

However, because of unbounded gradient at the margin, thickness errors decay slowly under grid refinement (Bueler and others, 2005). Thus, we measure the maximum and average thickness errors from both classical Mahaffy and $M^{\star}$ methods in Figure 6. Both methods show expected slow convergence of maximum error. The decay of the average error for $M^{\star}$, to $\sim 1 \mathrm{~m}$ for the three finest grids, is better than for classical Mahaffy. However, because of unbounded gradient at the margin, the measured $M^{\star}$ decay rate of $O\left(\Delta x^{1.47}\right)$ is less good than the theoretical $O\left(\Delta x^{2}\right)$ convergence rate expected from truncation-error analysis.

Improved convergence of the Newton iteration for the $M^{\star}$ scheme, which in this flat-bed case differs from the classical scheme only by improved quadrature, is seen in all cases. As indicated in Figure 6 by gray circles, Newton iteration for the classical Mahaffy scheme fails to converge for all grids except the coarsest. Though the $M^{\star}$ Newton iteration fails to converge at the $\varepsilon_{12}=0$ stage on the two finest grids, these cases fully converge if the problem is changed to a backward Euler step (Eqn (40)) of duration 100.0 years (not shown). Figure 7 shows clear evidence of quadratic, or at least superlinear, convergence from the Newton solver, in runs where the relative tolerance (i.e. residual 2-norm reduction factor) is set to $10^{-10}$. That is, at each stage $\varepsilon_{i}$ of the continuation scheme, the computed residual shows the characteristic curved drop of quadratic convergence on such semi-log axes (Kelley, 2003).

To test performance on non-flat and non-smooth beds, we use a glacier-scale bedrock-step exact solution by Jarosch and others (2013). As shown in Figure 8, the exact thickness is discontinuous at the cliff, as it goes to zero on the uphill side and has a non-zero value on the downhill side. Note that our computation uses two horizontal dimensions, but it is constant in the $y$-direction (not shown).

Figure 8 shows results both from $M^{\star}$ calculations and additional calculations without upwinding $\left({ }^{\prime} \lambda=0^{\prime}\right)$ and using the maximum upwind that does not expand the stencil (' $\left.\lambda=1^{\prime}\right)$ (Eqn (30)). These results suggest why we have chosen $\lambda=1$ / 4 in $M^{\star}$. For $\lambda=0$ there are large errors on the downhill

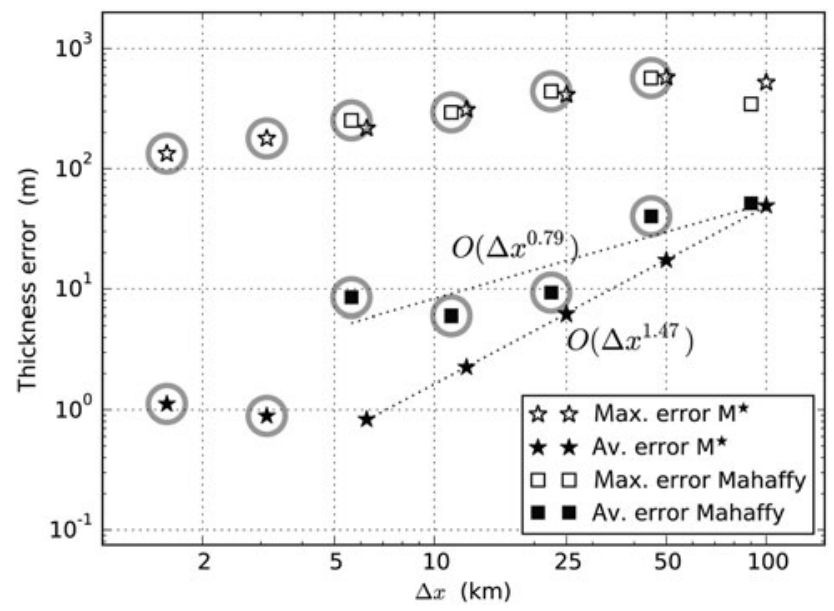

Fig. 6. Average and maximum error under grid refinement using the dome exact solution, for the $M^{\star}$ (stars) and classical Mahaffy (squares) methods. Gray circles indicate runs where the Newton method diverged before the last $\varepsilon_{12}=0$ continuation stage. 


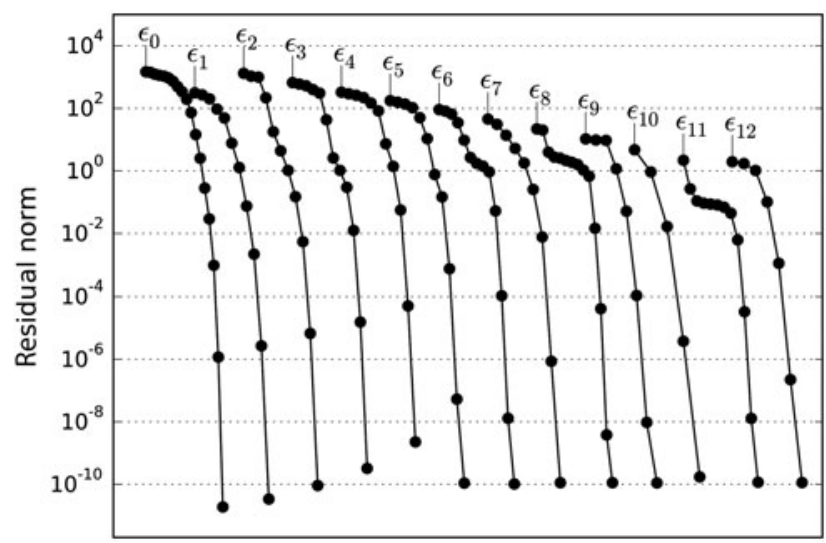

Fig. 7. Residual norm vs iteration number for each continuation stage. The SIA problem itself is the last $\varepsilon_{12}=0$ curve.

side of the cliff, while for $\lambda=1$ the uphill thickness is too large. Just a bit of upwinding captures the flux at the cliff, so that both uphill and downhill thicknesses are good.

To compare with results of Jarosch and others (2013), we applied the $M^{\star}$ method on grids with $\Delta x=\Delta y=1000,500$, 250, $125 \mathrm{~m}$. When we measure maximum thickness errors (not shown), there is no evidence of convergence as the grid is refined. However, maximum errors are not expected to decay. (This is because merely interpolating a discontinuous function like the exact solution with piecewise-linear functions generates large errors in the maximum norm.) For average thickness errors the evidence of convergence is unconvincing (not shown). The standard theory of FE methods also does not ensure convergence in average error, because of the extremely low regularity of the exact solution (Elman and others, 2005). In any case, error norms are not reported by Jarosch and others (2013), so we cannot compare with that source.

However, like Jarosch and others (2013) we can examine relative volume error, a weak quality measure, in addition to the visual evidence from Figure 8. Table 1 shows the value

$$
100 \frac{V_{\text {numerical }}-V_{\text {exact }}}{V_{\text {exact }}} .
$$

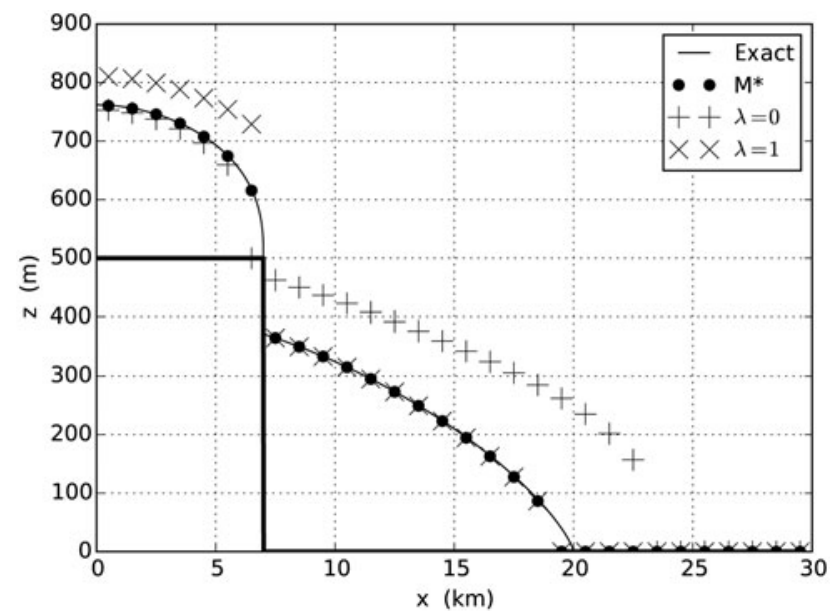

Fig. 8. Results from $M^{\star}$ method, and its upwinding variations with different $\lambda$ values in formula (30), compared with the bedrock-step exact solution, on a $\Delta x=1000 \mathrm{~m}$ grid. The bedrock itself is shown with a thick solid line.
The second, third and fourth columns are from the same three versions of the $M^{\star}$ method shown in Figure 8. Again we see why $\lambda=1 / 4$ is preferred to the upwinding alternatives. The last two columns of Table 1 show the results reported by Jarosch and others (2013) for their best 'Superbee'-limited MUSCL scheme and for their implementation of the classical Mahaffy scheme ('M2'). Thus, we see that results from our implicit, first-order-upwinding $M^{\star}$ scheme are highly satisfactory in this case.

\subsection{Greenland: bed topography and robustness}

While we have demonstrated its effectiveness on verification cases, the method's success ultimately depends on robust convergence when the data of the problem, especially the bed topography $b$, are realistically rough. To test robustness and high-resolution scalability we use two Greenland ice sheet bed topography datasets of different smoothness. We see that runs at high resolution (600-2000 m) converge only imperfectly on the rougher data, but that implicit time steps can be used to get arbitrarily close to steady state in such cases.

The smoother and older BEDMAP1 bed data are on a $5 \mathrm{~km}$ grid (Bamber and others, 2001); we call it 'BM1'. Along with a gridded model for present-day surface mass balance (Ettema and others, 2009), which all of our experiments use, it is included in the SeaRISE data (Bindschadler and others, 2013). The newer, finer-resolution, and rougher-bed data, on a $150 \mathrm{~m}$ grid, are from Morlighem and others (2014). This dataset, which we call ' $\mathrm{MCB}$ ', is generated by mass-conservation methods using both recent surface velocity measurements and a larger collection of ice-penetrating radar flightlines than were used for BM1.

Considering the $\mathrm{BM} 1$ bed first, we solved the problem on 5000, 2500, 1667, 1250, 1000 and $625 \mathrm{~m}$ grids. In the sub- $5 \mathrm{~km}$ cases, we refined the data using bilinear interpolation, and thus the bed became smoother under grid refinement. Figure 9 shows that, though the Newton solver does not converge at the final $\varepsilon_{12}=0$ level, the next-best level $\varepsilon_{11}$ is reached by the continuation scheme using the RS solver (see below) on all of the finer $(<2000 \mathrm{~m}$ ) grids. (It is unlikely that the slightly regularized SIA model at the $\varepsilon_{11}$ continuation level has any deficiencies whatsoever, as a model of real ice dynamics, relative to the unmodified $\varepsilon_{12}=0$ SIA model.)

The runs using the MCB data were on 4500, 3000, 1500, 1200,900 and $600 \mathrm{~m}$ grids. The bed elevations were averaged versions of the original $150 \mathrm{~m}$ postings, namely onto $30 \times 30$ blocks for the $9 \mathrm{~km}$ grid down to $4 \times 4$ blocks for the $600 \mathrm{~m}$ grid. Because this process reveals more and more detail, the bed became rougher under grid refinement. Except on the coarsest and most-averaged grid, the continuation scheme fails to generate convergent Newton solutions beyond the middle stages $\left(\varepsilon_{4}-\varepsilon_{6}\right)$. Thus, it is clear that highly resolved bed, which causes large and irregular values of $D$ and $\mathbf{W}$ to arise from formulas (3) and (6), respectively, limits the success of our combined continuation scheme and Newton iteration.

Though theoretical guidance on the choice of a solver for complementarity problems is, to our knowledge, lacking in this context, these Greenland datasets are realistic and challenging cases on which to compare the two available methods for solving Eqn (32). From Figure 9 it is clear that the convergence of the RS and SS methods (Benson and 
Table 1. Relative volume difference percentages Eqn (41) on the bedrock-step exact solution. ' $M^{\star \prime}$, ' $\lambda=0^{\prime}$ and ' $\lambda=1^{\prime}$ columns show the same three upwinding variations as in Figure 8. 'NC' indicates a Newton-iteration convergence failure. 'Superbee' and 'M2' columns are from Jarosch and others (2013)

\begin{tabular}{|c|c|c|c|c|c|}
\hline $\begin{array}{l}\Delta x \\
\mathrm{~m}\end{array}$ & $M^{\star}$ & $\lambda=0$ & $\lambda=1$ & Superbee & M2 \\
\hline 1000 & -1.205 & 49.067 & 9.217 & -7.588 & 116.912 \\
\hline 500 & 0.373 & 60.826 & 10.423 & -5.075 & 132.095 \\
\hline 250 & 1.220 & $\mathrm{NC}$ & 11.104 & -3.401 & 139.384 \\
\hline 125 & 1.621 & NC & 11.424 & -2.579 & 142.997 \\
\hline
\end{tabular}

Munson, 2006) is comparable, with the RS method slightly better in the high-resolution cases. However, the SS method is also three times slower on average over all runs in Figure 9.

Based on these initial experiments we implemented the following strategy for computing the steady-state solution using the MCB bed data averaged onto a $900 \mathrm{~m}$ grid and the RS solver: generate five smoothed versions of the bed data, with successively greater averaging before interpolating to the same $900 \mathrm{~m}$ grid; the least-smoothed of these data is the simple average of $6 \times 6$ blocks of the original $150 \mathrm{~m}$ data. At the first stage, compute the steady state using the most-smoothed version of the bed data, for which we expect convergence to a 'good' level (e.g. $\varepsilon_{i}<10^{-3}$ such as $\varepsilon_{10}-\varepsilon_{12}$ ). Extract the surface elevation from the result and construct a new initial thickness from it using the next less-smoothed version of the bed data. Though the steadystate continuation/Newton scheme will not converge from this new initial thickness, as convergence is quite insensitive to initial iterate, as it is blocked by bed roughness, one can expect a backward-Euler time step Eqn (40) to converge. Thus, we now start time-stepping. These implicit time steps are chosen sufficiently short so that the continuation scheme fully converges (i.e. to the $\varepsilon_{12}=0$ level), and thus we further approach steady state on the better bed. We iterate this bed-resolving strategy through the stages until using the least-smoothed bed, that is, the fully resolved bed on the $900 \mathrm{~m}$ grid. We can then continue time-stepping so as to approach steady state as closely as desired.

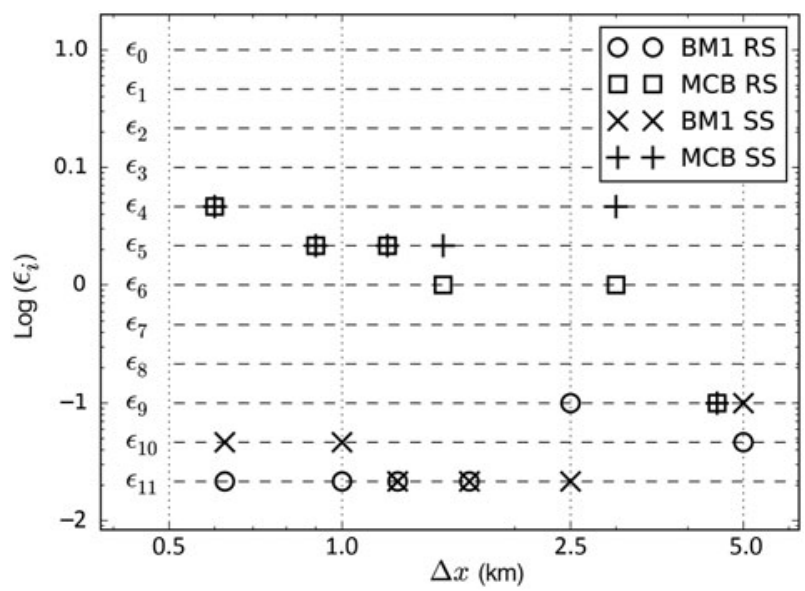

Fig. 9. Smallest successful value of $\varepsilon_{i}$ for which the Newton iteration converges on the steady-state problem; lower is better. Different bed data sources (BM1 or MCB) and complementarity-problem solvers (RS or SS) are compared.
The result of this strategy is shown in Figure 10. The first step was a nearly converged steady-state computation $\left(\varepsilon_{11}\right.$ level) on the most-smoothed bed. The five-step bed-resolving process in the last paragraph was applied using very short $\left(\sim 10^{-3}\right.$ years) backward Euler time steps. Then 50 model years of additional backward Euler time steps were run,

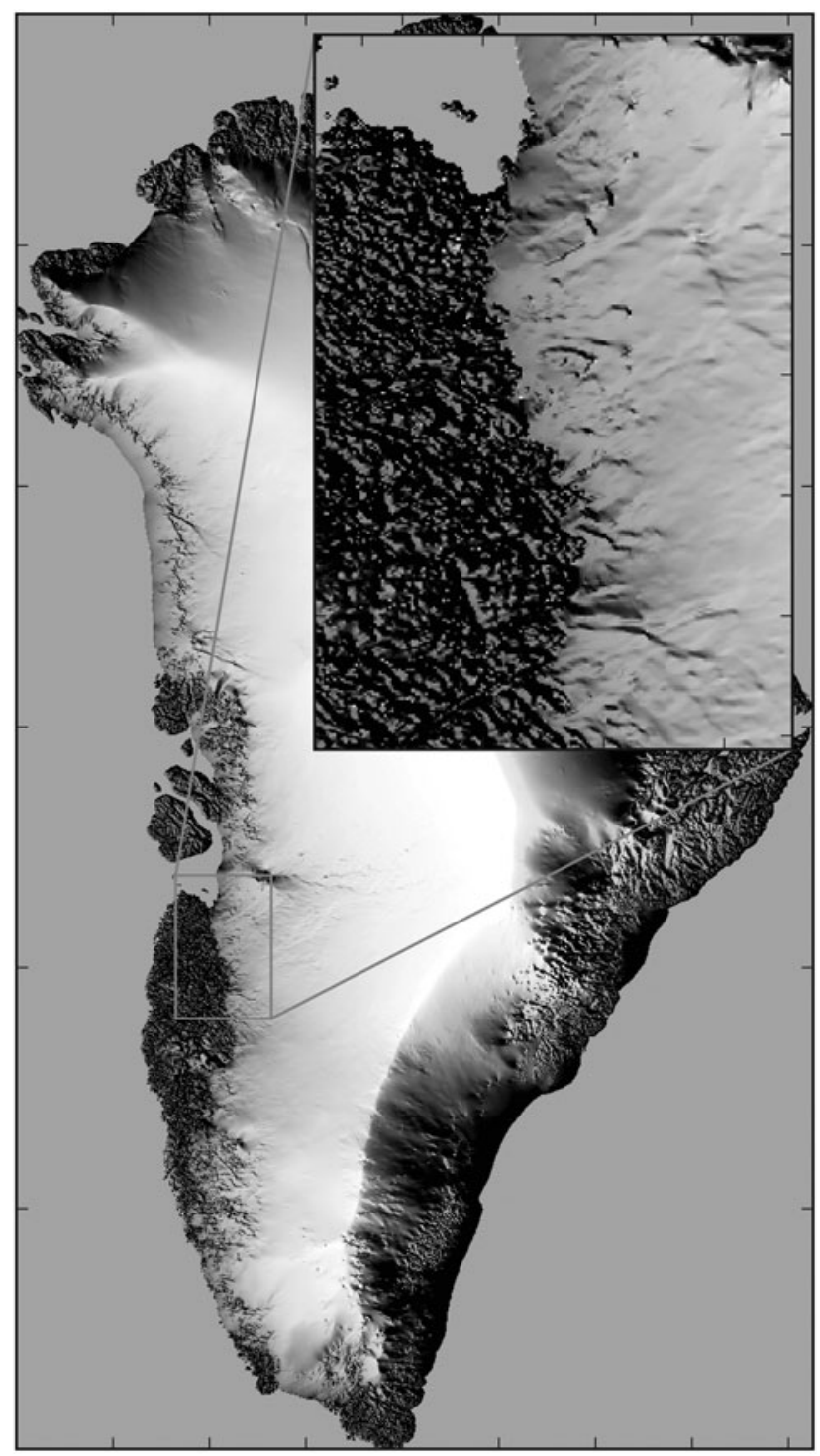

Fig. 10. Computed high-resolution ice sheet surface. The free boundary (margin) is determined only by the surface mass balance, bedrock topography and the steady-state, simplifieddynamics SIA model. We use $900 \mathrm{~m}$ model resolution and the MCB bed topography data. 
with 1 month time steps ( 0.1 years) for the last 20 years. The result in Figure 10 has volume $3.48 \times 10^{6} \mathrm{~km}^{3}$; compare the observed value, $2.95 \times 10^{6}$. The average absolute thickness error of $139 \mathrm{~m}$ is dominated by large errors from mislocating the ice margin in fjord-like coastal areas. Though the average diffusivity over the whole ice sheet was small $\left(D \approx 0.5 \mathrm{~m}^{2}\right.$ $\mathrm{s}^{-1}$ ), maximum values of $D \approx 6 \times 10^{3} \mathrm{~m}^{2} \mathrm{~s}^{-1}$ occurred in the interiors of highly resolved outlet glaciers.

\section{DISCUSSION AND CONCLUSION}

The problem solved in this paper is fundamentally different from most prior ice-sheet modeling. The steady-state geometry and extent of an ice sheet are computed directly as a function of only two datasets, namely the bed topography and surface mass balance. Though this function has not been proved to be well defined, even in the elevation-independent surface mass-balance cases here (compare Jouvet and others, 2011), there is theoretical support for wellposedness (Jouvet and Bueler, 2012) and nothing seen in our calculations suggests otherwise.

We do not claim that the model here, an untuned isothermal SIA model using EISMINT I parameter values, is sufficiently complete to represent all of the important physics of the Greenland ice sheet. By contrast, thermomechanically coupled shallow 'hybrid' models that include membrane stresses in the stress balance are indeed capable of veryhigh-quality match to the observations (Aschwanden and others, 2016).

Our numerical methods are based on both new and old ideas. The numerical discretization of the SIA equation starts from a classical structured-grid FD scheme (Mahaffy, 1976), but we make two improvements to create the new $M^{\star}$ scheme, both based on reinterpreting the classical scheme as an FVE method:

(1) improved quadrature in the flux integral, and

(2) first-order upwinding for the part of the ice flux which is proportional to the bed gradient.

Then, because the constraint of non-negative thickness makes this a free-boundary problem, we put it in nonlinear complementarity problem form and apply a parallel Newton solver designed for such problems.

In verification cases, the $M^{\star}$ method is both more accurate than, and gives substantially better Newton iteration convergence than, the classical scheme. We also show that the method can succeed at large scale and high resolution on real, highly irregular ice-sheet bed elevation data. Compared with the steady-state Greenland calculation by Jouvet and Bueler (2012), we have increased the number of unknowns by more than an order of magnitude, and we replaced a fixed-point iteration by a quadratically convergent Newton method.

Convergence of the Newton iteration in the presence of finely resolved, and thus very rough, bed is not guaranteed. Highly variable values of diffusivity $D$, such as those that arise in the computation that generated Figure 10 apparently form part of the barrier to full convergence of the continuation/Newton scheme for such steady-state computations. The convergence of long time steps on rough beds is also limited, but this was not studied quantitatively. A complete and/or precise understanding of the failure of convergence of the Newton iteration on rough beds is a topic for further research.

Though we have not tested it, the $M^{\star}$ ideas can be extended to unstructured dual Delaunay/Voronoi meshes such as those used by Egholm and Nielsen, (2010) and the MPAS Land Ice model (COSIM Team, 2013; Ringler and others, 2013). These models use $P^{1}$ FEs on a Delaunay triangulation and flux-integral quadrature on the Voronoi-cell edges. The improved quadrature idea (1) above would split the cell edges where the element (i.e. triangle) boundaries cross them, while the upwinding idea (2) requires interpretation as a first-order reconstruction after advection. Such a method would improve on that of Egholm and Nielsen (2010), in particular, by exploiting an underlying $P^{1}$ element flux approximation, instead of using a highly averaging and stencil-expanding least squares method.

The most important extension of our work, however, depends on noticing that it is actually rather flux-agnostic. In particular, the steady-state mass-conservation Eqn (7) or (8) also applies as stated with any Stokes (Greve and Blatter, 2009), 'higher-order' (Pattyn and others, 2008) or hybrid membrane-stress-resolving (Winkelmann and others, 2011) model that computes the ice-sheet geometry. In such models, the computation of the discrete flux $\mathbf{q}^{h}$ from the approximate ice-sheet thickness $H^{h}$ is completely different from the direct differentiation done here in the SIA, as it involves solving a separate elliptic-type stress balance model. For structured-grid models, however, the same basic FVE method (Eqns (26-28)) sets up the discrete equations. Then the problem becomes a version of complementarity problem (32), as ice thickness is non-negative regardless of the model for its stresses. Thereby one is faced with a free-boundary problem for the steady-state ice-sheet geometry. An adapted Newton solver is the natural choice to solve such problems; the residual evaluation in such cases always includes some kind of computation of $\mathbf{q}^{h}$ from $H^{h}$, however complicated. While the roughness of the bed is also a difficulty in solving such 'higher-order' stress-balance problems (Brown and others, 2013), the spatial integration used in all membrane-stress or full-stress resolving models should actually smooth the residual function seen by the Newton solver. To our knowledge, however, direct solution of steady-state Eqn (7) has not been attempted for other than SIA fluxes. Clearly, these are topics for further research.

\section{ACKNOWLEDGEMENTS}

Thanks to Scientific Editor R. Greve and reviewers A. Jarosch and G. Jouvet for helpful comments, which improved the presentation and content. Thanks also to B. Smith of Argonne National Laboratory for key help on the PETSc solvers. This work was supported by NASA grant \#NNX13AM16G and a grant of high-performance computing resources from the Arctic Region Supercomputing Center.

\section{REFERENCES}

Aschwanden A, Fahnestock MA and Truffer M (2016) Complex Greenland outlet glacier flow captured. Nat. Commun., 7 (doi: 10.1038/ncomms10524) 
Balay S and others (2015) PETSC users manual-revision 3.6. (Technical Report ANL-95/11) Argonne National Laboratory

Bamber J, Layberry R and Gogenini S (2001) A new ice thickness and bed data set for the Greenland Ice Sheet 1: measurement, data reduction, and errors. J. Geophys. Res., 106(D24), 33773-33780

Benson S and Munson T (2006) Flexible complementarity solvers for large-scale applications. Optim. Method. Softw., 21(1), 155-168

Bindschadler RA and 27 others (2013) Ice-sheet model sensitivities to environmental forcing and their use in projecting future sealevel (The SeaRISE project). J. Glaciol., 59(214), 195-224

Briggs W, Henson VE and McCormick S (2000) A multigrid tutorial, 2nd edn. SIAM Press

Brown J, Smith B and Ahmadia A (2013) Achieving textbook multigrid efficiency for hydrostatic ice sheet flow. SIAM J. SCi. Comput., 35(2), B359-B375 (doi: 10.1137/110834512)

Bueler E (2003) Construction of steady state solutions for isothermal shallow ice sheets. (Department of Mathematical Sciences Tech. Rep. 03-02) University of Alaska, Fairbanks

Bueler E and Brown J (2009) Shallow shelf approximation as a "sliding law" in a thermodynamically coupled ice sheet model. J. Geophys. Res., 114, F03008 (doi: 10.1029/2008JF001179)

Bueler E, Lingle CS, Kallen-Brown JA, Covey DN and Bowman LN (2005) Exact solutions and verification of numerical models for isothermal ice sheets. J. Glaciol., 51(173), 291-306

Cai Z (1990) On the finite volume element method. Numerische Mathematik, 58(1), 713-735 (doi: 10.1007/BF01385651)

Calvo N, Durany J and Vázquez C (2000) Numerical computation of ice sheet profiles with free boundary models. Appl. Numer. Math., 35(2), 111-128 (doi: http://dx.doi.org/10.1016/S01689274(99)00052-5)

Calvo N, Díaz J, Durany J, Schiavi E and Vázquez C (2002) On a doubly nonlinear parabolic obstacle problem modelling ice sheet dynamics. SIAM J. Appl. Math., 63(2), 683-707 (doi: 10.1137/S0036139901385345)

Ciarlet PG (2002) The finite element method for elliptic problems. SIAM Press, reprint of the 1978 original

COSIM Team (2013) MPAS-land ice model user's guide version 3.0. Los Alamos National Laboratory, http://mpas-dev.github.io/

Curtis A, Powell MJ and Reid JK (1974) On the estimation of sparse Jacobian matrices. J. Inst. Math. Appl., 13(1), 117-120

Egholm D and Nielsen S (2010) An adaptive finite volume solver for ice sheets and glaciers. J. Geophys. Res.: Earth Surface, 115(F1) (doi: 10.1029/2009JF001394)

Elman HC, Silvester DJ and Wathen AJ (2005) Finite elements and fast iterative solvers: with applications in incompressible fluid dynamics. Oxford University Press

Ettema J and 6 others (2009) Higher surface mass balance of the Greenland ice sheet revealed by high-resolution climate modeling. Geophys. Res. Lett., 36, L12501 (doi: 10.1029/ 2009GL038110)

Ewing RE, Lin T and Lin $Y$ (2002) On the accuracy of the finite volume element method based on piecewise linear polynomials. SIAM J. Numer. Anal., 39(6), 1865-1888

Fowler AC (1997) Mathematical models in the applied sciences. Cambridge University Press

Greve R and Blatter H (2009) Dynamics of ice sheets and glaciers. Advances in Geophysical and Environmental Mechanics and Mathematics, Springer

Hindmarsh RCA and Payne AJ (1996) Time-step limits for stable solutions of the ice-sheet equation. Ann. Glaciol., 23, 74-85

Hindmarsh RCA, Morland LW, Boulton GS and Hutter K (1987) The unsteady plane flow of ice-sheets: a parabolic problem with two moving boundaries. Geophys. Astrophys. Fluid Dyn., 39(3), 183-225 (doi: 10.1080/03091928708208812)

Hutter K (1983) Theoretical glaciology. D. Reidel

Huybrechts P and others (1996) The EISMINT benchmarks for testing ice-sheet models. Ann. Glaciol., 23, 1-12

Jarosch AH, Schoof CG and Anslow FS (2013) Restoring mass conservation to shallow ice flow models over complex terrain. Cryosphere, 7(1), 229-240 (doi: 10.5194/tc-7-229-2013)
Jouvet G and Bueler E (2012) Steady, shallow ice sheets as obstacle problems: well-posedness and finite element approximation. SIAM J. Appl. Math., 72(4), 1292-1314 (doi: 10.1137/ 110856654)

Jouvet G and Gräser C (2013) An adaptive Newton multigrid method for a model of marine ice sheets. J. Comput. Phys., 252, 419-437 (doi: 10.1016/j.jcp.2013.06.032)

Jouvet G, Rappaz J, Bueler E and Blatter H (2011) Existence and stability of steady state solutions of the shallow ice sheet equation by an energy minimization approach. J. Glaciol., 57(202), 345354

Kelley C (2003) Solving nonlinear equations with Newton's method. SIAM Press

Kinderlehrer D and Stampacchia G (1980) An introduction to variational inequalities and their applications. Pure and Applied Mathematics, Academic Press

LeVeque RJ (2002) Finite volume methods for hyperbolic problems. Cambridge Texts in Applied Mathematics, Cambridge University Press

Mahaffy MW (1976) A three-dimensional numerical model of ice sheets: tests on the Barnes Ice Cap, Northwest Territories. J. Geophys. Res., 81(6), 1059-1066

Morlighem M, Rignot E, Mouginot J, Seroussi H and Larour E (2014) Deeply incised submarine glacial valleys beneath the Greenland Ice Sheet. Nature Geosci., 7, 418-422 (doi: 10.1038/ngeo2167)

Morton KW and Mayers DF (2005) Numerical solutions of partial differential equations: an introduction, 2nd edn. Cambridge University Press

Pattyn F and 20 others (2008) Benchmark experiments for higherorder and full Stokes ice sheet models (ISMIP-HOM). Cryosphere, 2, 95-108

Ringler T, Petersen M, Higdon R, Jacobsen D, Jones $\mathrm{P}$ and Maltrud $\mathrm{M}$ (2013) A multi-resolution approach to global ocean modeling. Ocean Model., 69, 211-232

Smith B, Bjorstad P and Gropp W (1996) Domain decomposition: parallel multilevel methods for elliptic partial differential equations. Cambridge University Press

Strang G (1972) Variational crimes in the finite element method. In The mathematical foundations of the finite element method with applications to partial differential equations. Academic Press, 689-710

van der Veen CJ (2013) Fundamentals of glacier dynamics, 2nd edn. CRC Press

Winkelmann R and 6 others (2011) The Potsdam Parallel Ice Sheet Model (PISM-PIK) Part 1: model description. Cryosphere, 5, $715-726$

\section{APPENDIX \\ ANALYTICAL JACOBIAN}

To sketch the calculation of the analytical Jacobian for the $M^{\star}$ method, we first recall that each Eqn (31) comes from Eqn (26),

$$
F_{j, k}=\sum_{s=0}^{7} \mathbf{c}_{s} \cdot \mathbf{q}^{h}\left(x_{j}^{s}, y_{k}^{s}\right)-m_{j, k} \Delta x \Delta y .
$$

As the stencil of the $M^{\star}$ scheme is the nine-node box shown in Figure $1 \mathrm{~b}$, each row of the Jacobian has nine non-zero entries corresponding to locations $p, q$ where $F_{j, k}$ depends on $H_{p, q}$. We compute

$$
\frac{\partial F_{j, k}}{\partial H_{p, q}}=\sum_{s=0}^{7} \mathbf{c}_{s} \cdot \frac{\partial \mathbf{q}^{h}\left(x_{j}^{s}, y_{k}^{s}\right)}{\partial H_{p, q}},
$$


noting that $\partial \mathbf{q}^{h}\left(x_{j}^{s}, y_{k}^{s}\right) / \partial H_{p, q}$ is non-zero only if $H_{p, q}$ is one of the four nodal values on the element $\square_{u, v}$ containing the quadrature point $\left(x_{j}^{s}, y_{k}^{s}\right)$. Using index $\ell=0,1,2,3$ for the corners of rectangle $\square_{u, v}$, we need to write code to compute

$$
\mathbf{Q}_{\ell}^{s}=\frac{\partial \mathbf{q}^{h}\left(x_{j}^{s}, y_{k}^{s}\right)}{\partial H_{\ell}}
$$

when $\left(x_{j}^{s}, y_{k}^{s}\right)$ is in $\square_{u, v}$.

Derivatives are easiest to compute, in a $Q^{1} \mathrm{FE}$ method, using local coordinates $\xi=\left(x-x_{u}\right) / \Delta x$ and $\eta=\left(y-y_{v}\right) / \Delta y$ on $\square_{u, v}$, so that $0 \leq \xi, \eta \leq 1$. Bilinear interpolation defines a function $H_{u, v}=H_{u, v}(\xi, \eta)$ on $\square_{u, v}$ from interpolation of the four nodal values $H_{\ell,}$ and bed elevation function $b_{u, v}$ is similarly defined. Differentiation with respect to $\xi$ and $\eta$ gives vector-valued functions $(\nabla H)_{u, v}$ and $(\nabla b)_{u, v}$. Differentiation with respect to $H_{\ell}$ gives the scalar function $\partial H_{u, v} / \partial H_{\ell}$ and vector-valued function $\partial(\nabla H)_{u, v} / \partial H_{\ell}$. We write code for each of these $(\xi, \eta)$-dependent functions on $\square_{u, v}$. Then we write code to compute functions $D_{u, v}$ and $\partial D_{u, v} / \partial H_{\ell}$ from Eqn (3), and then $\mathbf{W}_{u, v}$ and $\partial \mathbf{W}_{u, v} / \partial H_{\ell}$ from Eqn (6), in local coordinates $\xi, \eta$ on $\square_{u, v}$.
Denote local coordinates of the quadrature point $\left(x_{j}^{s}, y_{k}^{s}\right)$ on element $\square_{u, v}$ by $\xi^{s}=\left(x_{j}^{s}-x_{u}\right) / \Delta x$ and $\eta^{s}=\left(y_{k}^{s}-y_{v}\right) / \Delta y$. Note that for each quadrature point, as in Figure 4, upwinding determines an additional evaluation point, whose local coordinates are denoted $\left(\xi_{\text {up }}^{s}, \eta_{\text {up }}^{s}\right)$. In these terms, from Eqn (6), for the evaluation of the residual, Eqn (A1), and for the evaluation of the Jacobian entries, Eqn (A2), we write code to compute

$$
\begin{aligned}
\mathbf{q}^{h}\left(x_{j}^{s}, y_{k}^{s}\right)= & -D_{u, v}\left(\xi^{s}, \eta^{s}\right)(\nabla H)_{u, v}\left(\xi^{s}, \eta^{s}\right) \\
& +\mathbf{W}_{u, v}\left(\xi^{s}, \eta^{s}\right) H_{u, v}\left(\xi_{\text {up }}^{s}, \eta_{\text {up }}^{s}\right)^{n+2}, \\
\mathbf{Q}_{\ell}^{s}=- & \frac{\partial D_{u, v}}{\partial H_{\ell}}\left(\xi^{s}, \eta^{s}\right)(\nabla H)_{u, v}\left(\xi^{s}, \eta^{s}\right) \\
- & D_{u, v}\left(\xi^{s}, \eta^{s}\right) \frac{\partial(\nabla H)_{u, v}}{\partial H_{\ell}}\left(\xi^{s}, \eta^{s}\right) \\
+ & \frac{\partial \mathbf{W}_{u, v}}{\partial H_{\ell}}\left(\xi^{s}, \eta^{s}\right) H_{u, v}\left(\xi_{\text {up }}^{s}, \eta_{\text {up }}^{s}\right)^{n+2} \\
+ & (n+2) \mathbf{W}_{u, v}\left(\xi^{s}, \eta^{s}\right) H_{u, v}\left(\xi_{\text {up }}^{s}, \eta_{\text {up }}^{s}\right)^{n+1} \\
& \cdot \frac{\partial(\nabla H)_{u, v}}{\partial H_{\ell}}\left(\xi_{\text {up }}^{s}, \eta_{\text {up }}^{s}\right) .
\end{aligned}
$$

MS received 17 May 2015 and accepted in revised form 16 October 2015; first published online 18 March 2016 